\title{
Le zonage ou la spatialisation des fonctions de la réserve de biosphère du Ferlo (Nord-Sénégal)
}

\author{
Daouda NGOM ${ }^{1 *}$, Olimata FAYE ${ }^{2}$, Nouhou DIABY ${ }^{3}$ et L. E. AKPO ${ }^{4}$ \\ ${ }^{1}$ Université de Ziguinchor, Département d'Agroforesterie, Sénégal. \\ ${ }^{2}$ Direction des Eaux, Forêts et Chasses, Ministère en charge de l'Environnement, Sénégal. \\ ${ }^{3}$ IFAN / Université Cheikh Anta Diop de Dakar, Sénégal. \\ ${ }^{4}$ Laboratoire d'Ecologie et d'Ecohydrologie, Université Cheikh Anta Diop de Dakar, Sénégal. \\ *Auteur correspondant; E-mail : ngom_daouda@yahoo.fr; Tél :00 (221) 776571537
}

\section{RESUMÉ}

Afin de combiner efficacement conservation, utilisation durable des ressources et production du savoir, la réserve de biosphère doit nécessairement bénéficier d'une stratification, d'un zonage intégré et d'une gestion coopérative. La méthode de zonage est basée sur une caractérisation des ressources végétales, suivie de la spatialisation des enjeux et du zonage de la réserve de biosphère. Pour être partagé avec les populations locales, le zonage de la réserve de biosphère du Ferlo (RBF) a procédé par des réunions d'information et de sensibilisation, des observations sur le terrain, des enquêtes et une collecte de données biophysiques. L'étude des paramètres structuraux de la végétation a révélé que l'aire centrale de la RBF présente une densité d'arbres et un potentiel de régénération plus élevés. L'analyse du spectre d'abondance a montré que Guiera senegalensis J.F. Gmel est l'espèce la plus abondante dans la réserve. L'indice de diversité a révélé que la zone tampon et l'aire de transition qui font l'objet de multiples usages et qui subissent l'action de l'homme, présentent une diversité plus grande et un niveau d'organisation du peuplement ligneux plus élevé que l'aire centrale qui est une zone de conservation intégrale. Le traitement des images satellitales et la photointerprétation ont permis d'élaborer une carte d'occupation des sols et une carte globale du zonage. Ce zonage participatif a établi une spatialisation des fonctions de la réserve de biosphère sans pour autant les superposer. (C) 2012 International Formulae Group. All rights reserved.

Mots clés: Réserve de biosphère, carte d'occupation, zonage, conservation, biodiversité, utilisation du râble.

\section{INTRODUCTION}

Les réserves de biosphère sont des aires portant sur des écosystèmes terrestres et côtiers/marins qui visent à promouvoir des solutions pour réconcilier la conservation de la biodiversité avec son utilisation durable (UNESCO, 1996). Les réserves de biosphère doivent répondre, en effet, à trois fonctions majeures, qui se complètent et se renforcent mutuellement: la fonction de conservation de la diversité biologique, la fonction de développement et l'appui logistique ou la production de savoir. Elles doivent disposer, pour remplir leurs multiples fonctions, d'un zonage qui est une stratification du territoire par la spatialisation des usages dans l'espace. Le zonage distingue dans chaque réserve de biosphère une ou plusieurs aires centrales, une zone tampon bien identifiée et une aire de transition flexible. Pour relever les nouveaux défis qui se posent, il importe d'adopter un zonage plus intégré. Ainsi, l'aire de transition, 
outre sa fonction de développement, peut répondre à des objectifs environnementaux de conservation, et l'aire centrale, outre sa fonction de conservation, contribuer à une série de services d'écosystèmes chiffrables, du point de vue du développement, en termes économiques (séquestration du carbone, stabilisation des sols, qualité de l'air et de l'eau, etc.). De même, si l'éducation, la recherche, la surveillance continue et le renforcement des capacités sont vus comme des composantes de la fonction de support logistique ou de production de savoir, ils s'inscrivent aussi dans les fonctions de conservation et de développement (UNESCO, 2008).

Dans le processus de mise en place d'une réserve de biosphère le zonage apparait comme une spatialisation intégrée des différentes fonctions. Ainsi, la cartographie doit être réalisée de façon participative et inclusive avec l'appui et la collaboration des populations locales. Il constitue un outil indispensable pour concilier dans un même espace la conservation de la diversité biologique et l'utilisation rationnelle des ressources naturelles par les communautés locales (UNESCO, 2008).

La présente étude s'est fixé comme objectif d'établir un zonage inclusif et fonctionnel de la réserve de biosphère du Ferlo. Il s'agira de caractériser les ressources végétales, d'organiser des réunions d'information et de sensibilisation, de procéder à des observations de terrain, et d'élaborer les cartes d'occupation des sols et de zonage de la RBF.

\section{MATERIEL ET METHODES \\ La zone d'étude}

L'étude a été menée dans la réserve de biosphère du Ferlo (RBF) située au nord-est du Sénégal entre $14^{\circ} 24^{\prime}-16^{\circ} 11^{\prime}$ de latitude N et $13^{\circ} 07^{\prime}-14^{\circ} 51$ ' de longitude W (Figure 1). Elle est à cheval sur les régions administratives de Matam, Louga et SaintLouis (partie grisée). Plusieurs groupes ethniques (Peuls, Wolofs, Maures) cohabitent dans cet espace avec une prédominance des Peuls.

Le Ferlo est établi sur des formations sableuses dunaires du continental terminal. Le relief, peu accentué avec des pentes inférieures à 3\% (Cornet, 1992) joue un rôle dans l'évolution des milieux. Il permet de distinguer trois principaux types de sols : les sols sableux, les sols argilo-sableux à argileux et les sols latéritiques cuirassés.

Le climat est tropical sec, de type soudano-sahélien, caractérisé par une saison des pluies de 2 ou 3 mois et des amplitudes thermiques élevées. La température moyenne annuelle s'établit à $28,6^{\circ}$. Les valeurs moyennes des températures minimales et maximales mensuelles sont respectivement de $17^{\circ} \mathrm{C}$ (décembre) et $43{ }^{\circ} \mathrm{C}$ (mai).

Les précipitations sont concentrées en juillet, août et septembre. La pluviométrie moyenne de la station de référence de Ranerou est de 474 mm (1951 à 2011). Depuis 1970, plus de $60 \%$ des années présentent des pluviométries inférieures à la moyenne interannuelle; ce sont des années sèches (Figure 2).

D'un point de vue phytogéographique, la réserve de biosphère est à cheval sur les formations sahéliennes et les savanes soudaniennes. La strate ligneuse est à dominante arbustive; elle est composée de Guiera senegalensis J. F. Gmel., Pterocarpus lucens Lepr. Ex Guill. et Perrott., Combretum glutinosum Perrott. ex DC., Boscia senegalensis (Pers.) Lam. Ex Poir., Grewia bicolor Juss., Acacia senegal (L.) Willd et Balanites aegyptiaca (L.) Del. , etc. La strate herbacée est dominée par des graminées légères dont les plus communes sont Schoenefeldia gracilis Kunth., Andropogon pseudapricus Stapf., Pennisetum pedicellatum Trin., Eragrostis tremula Hochst., Cenchrus biflorus Roxb., Dactyloctenium aegyptium Beauv.

\section{Réunions d'information et de sensibilisation}

Des rencontres d'information et de sensibilisation des populations environnantes 
de la réserve de biosphère ont été organisées. Des émissions radio animées par les services techniques de l'environnement ont permis d'informer les populations du déroulement des activités de zonage afin que leur participation soit effective. Des réunions et des entrevues de groupe ont été organisées dans les villages polarisés par la réserve de biosphère. L'entrevue de groupe est un procédé qui s'est révélé particulièrement utile. En effet, elle permet en peu de temps d'avoir des éclaircissements sur la perception qu'ont les populations des limites des aires protégées avoisinantes.

\section{Caractérisation des ressources végétales}

Pour caractériser la végétation ligneuse, des relevés de végétation on été effectués dans le territoire de la réserve de biosphère. L'échantillonnage a utilisé la méthode des transects. Au total, sept transects d'orientation W-E de longueurs différentes et distant de 4 $\mathrm{km}$ ont été choisis. Les points de relevés de végétation ligneuse ont été choisis de manière aléatoire comme l'ont suggéré de nombreux auteurs tels que Gounot (1969) et Long (1974). Les points de relevés sont repérés sur le terrain à l'aide d'un GPS.
L'échantillon est de 110 relevés dans l'ensemble de la réserve de biosphère avec 57 relevés dans l'aire centrale, 28 en zone tampon et 25 dans l'aire de transition. L'unité d'échantillonnage est une placette carrée de 30 m X 30 m, soit une aire de relevée de 900 $\mathrm{m}^{2}$ (Boudet, 1984) pour l'étude de la végétation sahélo-soudanienne.

Dans chaque relevé, un recensement exhaustif des ligneux a été effectué. Des mensurations dendrométriques ont été réalisées pour évaluer quelques paramètres dimensionnels : la circonférence à la base du tronc à $30 \mathrm{~cm}$ du sol (Akpo et Grouzis, 1996), le diamètre de la projection du houppier au sol dans deux directions (nord-sud et est-ouest), la hauteur des arbres et la distance entre deux arbres par la méthode du plus proche individu. Les éléments topographiques, le sol et le substrat géologique du peuplement sont également relatés.

Les échantillons botaniques ont été identifiés sur le terrain ou en laboratoire à l'aide d'ouvrages notamment Flore $d u$ Sénégal (Berhaut, 1967) et Arbres, Arbustes et Lianes d'Afrique de l'Ouest (Arbonnier, 2000).

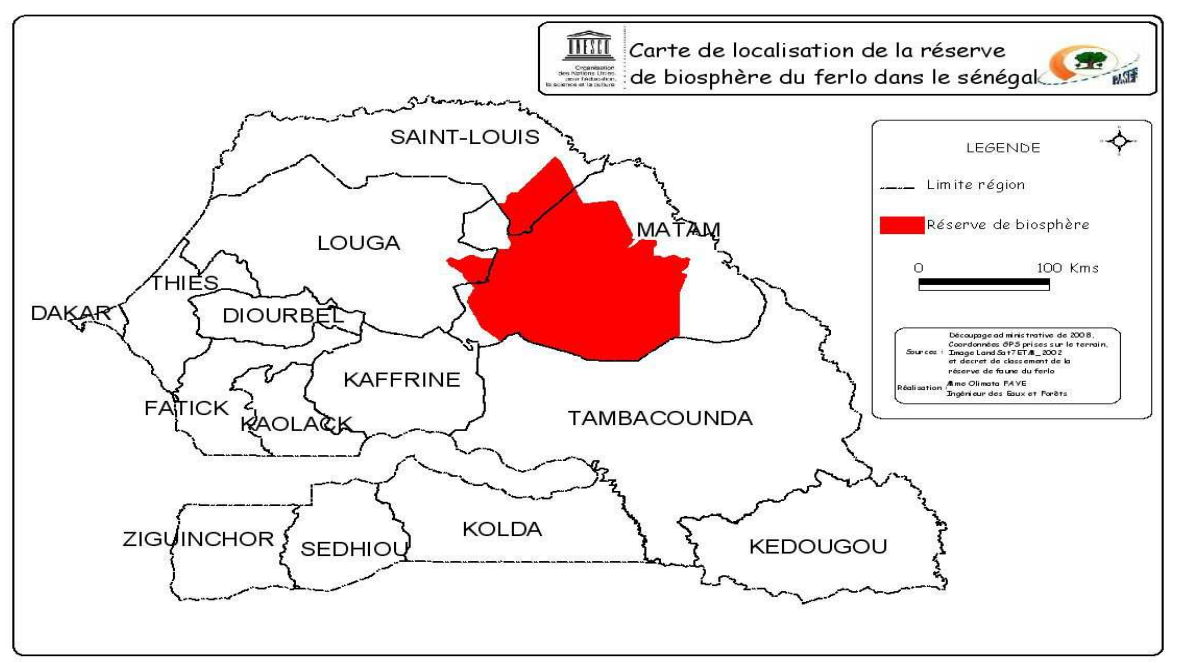

Figure 1: Carte de localisation de la réserve de biosphère du Ferlo. 


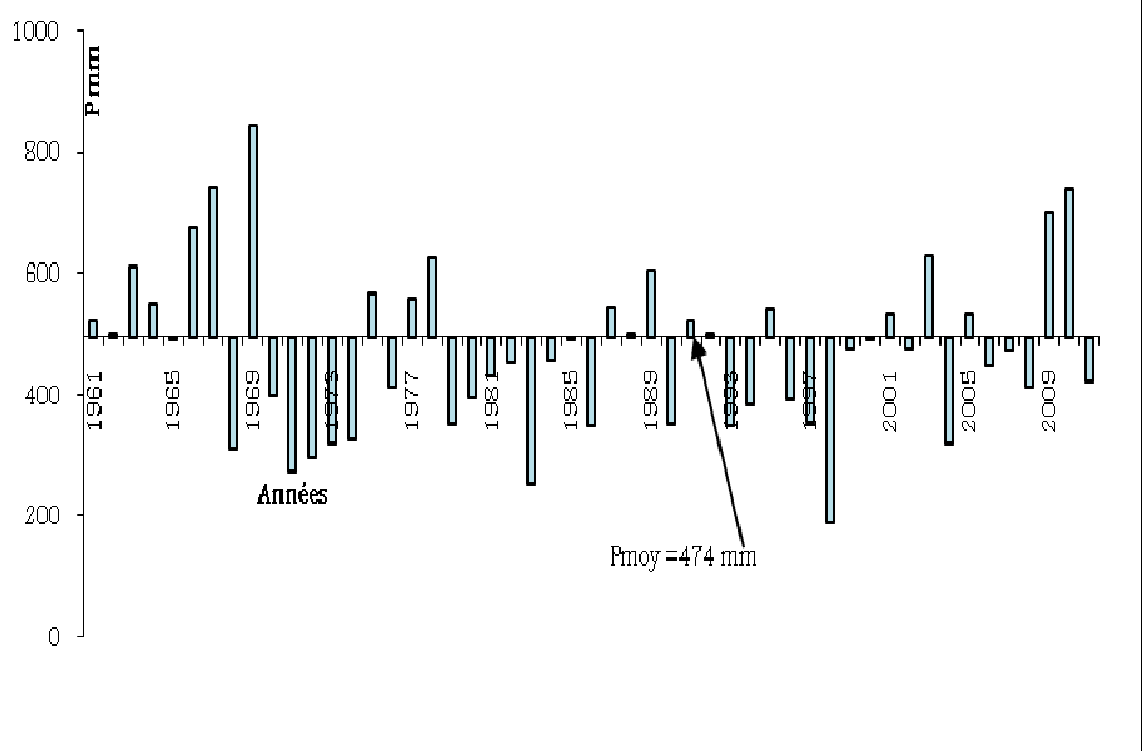

Figure 2: Tendances évolutives de la pluviosité annuelle par la méthode des moyennes mobiles pondérées.

\section{Délimitation des zones}

Dans le travail de délimitation des différentes zones de la réserve de biosphère, nous nous sommes appuyés sur la participation effective des communautés locales. Ce travail consiste à identifier et à géo-référencier les limites des différentes composantes de la réserve de biosphère. Elle a été faite à partir des levés GPS (Global Positioning System) en coordonnées UTM (Universal Transverse Mercator) suite à plusieurs rencontres avec les autorités et les populations locales.

L'identification des aires centrales de la réserve de biosphère a été faite selon l'importance écologique du site et l'absence d'établissements humains dans la zone considérée.

Nous avons ensuite procédé à l'inventaire et l'identification de groupes de villages polarisés par chaque noyau. Des pancartes de signalisation ont été installées dans les différents noyaux centraux délimités.
Par la suite, les zones tampon et de transition ont été identifiées et délimitées avec l'implication des populations locales.

\section{Le traitement des données \\ Traitement des données de végétation}

Les données collectées à partir des relevés de végétation ont été traitées grâce aux logiciels informatiques Excel, XLStat et Mintab. Ces logiciels nous ont permis d'étudier les paramètres structuraux de la végétation ligneuse notamment: $L a$ densité qui est le nombre d'individus par unité de surface. Elle s'exprime en nombre d'individus/ha. Le couvert ligneux qui est la surface de la couronne de l'arbre projetée verticalement au sol. Il est exprimé en mètre carré par hectare $\left(\mathrm{m}^{2} \cdot \mathrm{ha}^{-1}\right)$. Le couvert ligneux est calculé avec la formule ci-dessous :

$\mathrm{C}=\frac{\sum \pi\left(\frac{\mathrm{d} \mathrm{mh}}{2}\right)^{2}}{\mathrm{~S}_{\mathrm{E}}}-\operatorname{avec} C=$ couvert ligneux; $d_{m b}=$ diamètre moyen $\mathrm{du}$ 
houppier en $\mathrm{m}$; $S=$ surface de l'échantillon considéré en ha.

La surface terrière désigne la surface de l'arbre évaluée à la base du tronc de l'arbre. Elle est exprimée en mètre carré par hectare $\left(\mathrm{m}^{2} \cdot \mathrm{ha}^{-1}\right)$. Elle est donc obtenue à partir de la formule suivante :

$\mathrm{S}_{\mathrm{t}}=\frac{\sum \pi\left(\frac{\mathrm{d} 0,3}{2}\right)^{2}}{\mathrm{~S}_{\mathrm{E}}}$

Avec $S_{t}=$ surface terrière ; $d_{0,3}=$ diamètre en $\mathrm{m}$ du tronc à $0,3 \mathrm{~m} ; S_{E}=$ surface de l'échantillon considéré en ha.

\section{- L'abondance des espèces}

L'abondance est l'importance numérique relative d'une espèce dans un peuplement. Dans le cadre de cette étude, nous avons calculé la probabilité d'occurrence de l'espèce pi. Si dans un peuplement donné, $n i$ est le nombre d'individus d'une espèce $i$ et $N$ le nombre total d'individus que comporte le peuplement, on aura la formule suivante (Ramade 2002) :

$$
\text { pi }=\frac{\text { ni }}{\mathrm{N}}
$$

En ramenant la probabilité d'occurrence en pourcentage, nous avons la fréquence d'occurrence.

- L'indice de diversité Shannon-Weaver $\left(H^{\prime}\right)$ qui considère à la fois l'abondance et la richesse spécifique, convient bien à l'étude comparative des peuplements parce qu'il est relativement indépendant de la taille de l'échantillon (Ramade, 2003).

L'Indice de Shannon-Weaver, exprimé en bits, est donné par la formule suivante :

$$
\mathbf{H}^{\prime}=-\sum_{\mathrm{i}=1}^{\mathrm{S}} \frac{\mathrm{Ni}}{\mathrm{N}} \log 2 \frac{\mathrm{Ni}}{\mathrm{N}}
$$

avec $\mathrm{Ni}=$ l'effectif de l'espèce $\mathrm{i} ; \mathrm{N}=$ effectif total des espèces.

Cet indice $H^{\prime}$ est minimal si tous les individus du peuplement appartiennent à une seule et même espèce; il est maximal quand tous les individus sont répartis d'une façon égale sur toutes les espèces (Frontier, 1983). Dans la nature, quel que soit le groupe taxinomique étudié, les indices de diversité de Shannon sont compris entre 0 et 4,5 ; rarement davantage (Frontier et Pichod-Viale, 1998 ; Margalef, 1972 cité par Maguran, 1988).

- Le taux de régénération du peuplement est donné par le rapport en pourcentage entre l'effectif total des jeunes plants et l'effectif total du peuplement (Poupon, 1980) :

$$
T R P=\frac{\text { Effectif total des jeunes plants }}{\text { Effectif total du peuplement }} \times 100
$$

- L'Importance spécifique de régénération est quant à elle obtenue à partir du rapport en pourcentage entre l'effectif des jeunes plants d'une espèce et l'effectif de jeunes plants dénombrés (Akpo \& Grouzis, 1996) :

$$
I S R=\frac{\text { Effectif des jeunes plants d' une espèce }}{\text { Effectif total des jeunes plants dénombrés }} \times 100
$$

\section{Traitement des images satellitales}

L'image-interprétation est basée sur des images Landsat 7 ETM 2002 acquises par le Projet de Gestion Durable et Participative des Energies traditionnelles et de Substitution (PROGEDE). Ces images sont traitées au niveau «system corrected», ré-échantillonnées suivant la méthode du «nearest neighbour» en format «GeoTIFF» (Geographic Tag Image File Format), projetées dans le système UTM (projection Universelle Transverse de Mercator) sur l'ellipsoïde «WGS 84 » (World Global Spheroïd) au «datum» WGS 84. Elles possèdent une résolution au sol de $30 \mathrm{~m}$ (canaux 1 - bleu, 2 - vert, 3 - rouge, 4 - proche infrarouge, 5 - moyen infrarouge proche et 7 - moyen infrarouge lointain), de $60 \mathrm{~m}$ (canal 6 - thermique) et enfin de $15 \mathrm{~m}$ (canal 8 - panchromatique). La première opération a consisté à rassembler ces différents fichiers (GeoTIFF) au sein d'une image multilayer de type IMAGINE. Cette image multilayer est constituée de 6 bandes (canal ou layer) que sont : $1 ; 2 ; 3 ; 4 ; 5 ; 7$. La bande 8 constitue 
une image (noir et blanc) à part qui est la panchromatique. Toutes les images ont été reprojetées sur l'ellipsoïde de Clarke 1880 au «datum» Adindan Sénégal suivant une projection UTM, zone $28 \mathrm{P}$.

Les images ont été soumises à une classification non-supervisée en exploitant surtout les canaux 4 et 3 . La classification résultante (image thématique) a ensuite été recodée par regroupement des classes aux signatures spectrales similaires.

\section{Photo-interprétation et classification}

Après la classification sur l'ordinateur c'est-àdire basée sur la préfectance chlorophyllienne de la végétation, une mission de terrain a été organisée pour vérifier à quoi correspond chaque classe selon la classification des types africains de végétation de Yangambi (Aubreville, 1957).

\section{RESULTATS}

Les paramètres structuraux de la végétation ligneuse

Les relevés de végétation effectués dans les différentes unités de la réserve de biosphère ont permis de déterminer les différents paramètres structuraux de la végétation ligneuse (Tableau 1).

\section{La densité réelle}

Dans la RBF la densité réelle est de 389 pieds/hectare. Cependant, elle est plus élevée dans l'aire centrale (392) que dans la zone tampon (352) et l'aire de transition (347).

Les espèces qui présentent les plus fortes densités ont varié d'une zone à l'autre.

- Dans l'aire centrale : Guiera senegalensis, Pterocarpus lucens et Boscia senegalensis avec respectivement des densités de 154 ; 63 et 50 individus par hectare. Deux espèces (Guiera senegalensis et Pterocarpus lucens) représentent plus de la moitié de la densité moyenne de l'aire centrale (214 pieds/ha).

- Dans la zone tampon: Guiera senegalensis, Balanites aegyptiaca et Combretum glutinosum avec respectivement 76 pieds/ha, 47 pieds/ha et 45 pieds/ha.
- $\quad$ Dans l'aire de transition : Combretum glutinosum, Guiera senegalensis, Boscia senegalensis, avec respectivement 113 pieds/ha, 48 pieds/ha et 39 pieds/ha sont les espèces les plus présentes dans cette zone.

\section{Le recouvrement}

Le taux de recouvrement est de $35,3 \%$ dans l'ensemble de la réserve de biosphère. Il varie en fonction des différentes zones car sa valeur dépend fortement de la présence de grands arbres aux larges houppiers. Il est de $32 \%$ pour l'aire centrale, $43 \%$ en zone tampon et $35 \%$ dans l'aire de transition (Tableau 1).

Le taux de recouvrement des espèces dominantes dans les différentes zones de la RBF se présente comme suit :

- Dans l'aire centrale : Pterocarpus lucens (12,5\%), Guiera senegalensis (6\%), Combretum glutinosum $(2,9 \%)$ et Grewia bicolor $(1,7 \%)$.

- En zone de transition: Pterocarpus lucens (13,7\%), Guiera senegalensis (5,9\%), Combretum glutinosum (5,8\%) Acacia senegal (4\%) et Balanites senegalensis $(3,45 \%)$.

- Dans l'aire de transition: Combretum glutinosum (10,14\%) Pterocarpus lucens $(3,3 \%)$, Sterculia setigera $(3,1 \%)$, Guiera senegalensis $(3,1 \%)$ et Acacia senegal (2,8\%).

\section{La surface terrière}

La surface terrière moyenne de la RBF est de $7,44 \mathrm{~m}^{2} / \mathrm{ha}$. Elle varie de $6,1 \mathrm{~m}^{2} / \mathrm{ha}$ dans l'aire centrale à $9,17 \mathrm{~m}^{2} /$ ha en zone tampon et $8,64 \mathrm{~m}^{2} /$ ha dans l'aire de transition.

La surface terrière des espèces dominantes dans les différentes zones de la RBF se présente comme suit :

- Dans l'aire centrale Pterocarpus lucens (2,33 $\left.\mathrm{m}^{2} / \mathrm{ha}\right)$, Adansonia digitata $\left(1,25 \mathrm{~m}^{2} / \mathrm{ha}\right)$ et Guiera senegalensis $\left(0,45 \mathrm{~m}^{2} / \mathrm{ha}\right)$ présentent les surfaces terrières les plus élevées et contribuent pour près de $67 \%$ à la surface terrière de la réserve de biosphère.

- $\quad$ En zone tampon, Pterocarpus lucens $(4,3$ $\left.\mathrm{m}^{2} / \mathrm{ha}\right)$, Combretum glutinosum (1,03 $\left.\mathrm{m}^{2} / \mathrm{ha}\right)$ et Balanites aegyptiaca $\left(0,62 \quad \mathrm{~m}^{2} / \mathrm{ha}\right)$ contribuent pour $65 \%$ de la surface terrière. 
- Dans l'aire de transition, Combretum glutinosum $\left(2,1 \mathrm{~m}^{2} / \mathrm{ha}\right)$, Sterculia setigera (1,47 m²/ha), Adansonia digitata (1,08 m²/ha) dominent largement car elles représentent plus de la moitié de la surface terrière de la zone de transition.

\section{L'abondance spécifique}

La représentation graphique de la distribution d'abondance relative des espèces constituant la réserve de biosphère (Figure 3) montre que les différentes zones de la RBF présentent des différences notables d'effectifs des ligneux rencontrés.

Dans l'aire centrale, Guiera senegalensis est l'espèce la plus abondante (40\%), suivie de Pterocarpus lucens (16\%), Boscia senegalensis (13\%) et Combretum glutinosum (10\%). Ces quatre espèces représentent $80 \%$ des effectifs de l'unité. En zone tampon, Guiera senegalensis (21,8\%), Balanites aegyptiaca (13,4\%), Boscia senegalensis $(12,8 \%)$ et Combretum glutinosum $(11,4 \%)$ sont les quatre espèces les plus abondantes avec une fréquence d'occurrence totale de 60\%. Dans l'aire de transition, l'ordre d'abondance des espèces a changé par rapport aux deux autres zones. En effet, Combretum glutinosum qui était quatrième de par son abondance dans l'aire centrale et la zone tampon devient l'espèce la plus abondante dans l'aire de transition avec une fréquence d'occurrence de 32,7\%. Elle est suivie de Guiera senegalensis (13,8\%), Boscia senegalensis $(11,8 \%)$ et Balanites aegyptiaca $(7,9 \%)$.

Globalement, dans la réserve les quatre espèces les plus abondantes sont les mêmes que dans l'aire centrale avec une différence de rang. Il s'agit de Guiera senegalensis (29,5\%), Combretum glutinosum (15,9\%), Pterocarpus lucens $(11,6 \%)$ et Boscia senegalensis $(10,5 \%)$. Ces quatre espèces représentent $68 \%$ de l'ensemble des effectifs de la réserve de biosphère.

\section{L'indice de diversité de Shannon}

Pour mieux appréhender la diversité entre les différentes zones de la RBF, nous avons procédé par le calcul de l'indice de diversité le plus utilisé en Ecologie : l'indice de Shannon. L'analyse des résultats (Tableau 1) montre que l'indice de diversité de Shannon est assez élevé dans la réserve de biosphère avec une valeur de 3,45 bits. Il varie très peu dans les différentes zones avec 2,92 dans l'aire centrale à 3,55 en zone tampon et 3,49 dans l'aire de transition.

\section{La régénération naturelle du peuplement}

Dans la réserve de biosphère, la régénération du peuplement a été évaluée par l'importance des jeunes plants (circonférence $<10 \mathrm{~cm})$. Le taux de régénération du peuplement est de $72 \%$ dans l'ensemble de la RBF. Il varie fortement d'une zone à l'autre. Il est deux fois plus élevé dans l'aire centrale (79\%) qui est moins anthropisée que dans la zone tampon (36\%) et l'aire de transition (39\%) (Tableau 1).

L'importance de la régénération en fonction des différentes espèces a été appréhendée par le calcul de l'indice spécifique de régénération (ISR) qui a révélé que Guiera senegalensis a le meilleur potentiel de régénération de la $\mathrm{RBF}$ avec un indice spécifique de régénération de $62,37 \%$. Elle est suivie de Boscia senegalensis avec $16,38 \%$. Ces deux espèces comportent $78 \%$ des jeunes plants inventoriés dans la RBF.

\section{La carte d'occupation des sols}

La classification a fait ressortir les différentes strates suivantes:

\section{- la zone agricole}

Elle est localisée dans la partie Sud de la réserve et occupe une superficie de 36120 ha (soit $2 \%$ de la superficie de la réserve). Ce sont des cultures de cases ou vivrières qui $\mathrm{y}$ sont pratiquées. Les principales spéculations sont surtout le mil et le maïs. Le sorgho est également cultivé dans certaines zones dépressionnaires (Faye, 2011).

- zone à faible couverture végétale ligneuse correspondant essentiellement à des steppes arbustives à arborées et aux sols nus

Elle est localisée dans la partie nord de la réserve et occupe une superficie de 532107 
ha, soit $26 \%$ de la superficie totale de la réserve. Cette strate est constituée par les sols nus, les steppes arbustives à arborées, les mares, les habitations et certaines zones de cultures. Les espèces ligneuses dominantes sont Guiera senegalensis, Commiphora africana, et Boscia senegalensis. Au niveau des mares, on rencontre principalement Mitragyna inermis, Balanites aegyptiaca, Acacia seyal, Anogeissus leiocarpus,Ziziphus mauritiana, etc.

- zone à couverture végétale ligneuse peu riche correspondant essentiellement à des savanes arbustives

Cette strate qui s'étend sur 329575 ha, représente $16 \%$ de la superficie totale de la réserve de biosphère. Les espèces ligneuses dominantes sont Guiera senegalensis et Pterocarpus lucens généralement associées à Combretum glutinosum et Boscia senegalensis.

- zone à couverture végétale ligneuse riche correspondant essentiellement à des savanes arbustives à arborées et aux formations de vallée (galeries forestières)

Elle constitue la strate la plus importance avec $56 \%$ de la superficie; soit 1148700 ha. Elle se localise au centre et au sud de la réserve. Les espèces dominantes sont: Guiera senegalensis, Pterocarpus lucens, Combretum glutinosum et Grewia bicolor dans la savane arbustive à arborée et Bauhinia reticulata, Mytragina inermis, Acacia seyal, etc. dans les galeries forestières.

- zone à couverture végétale ligneuse très riche correspondant essentiellement aux savanes arborées à boisées

Elle se localise au sud de la réserve vers la région de Tambacounda. C'est la strate la moins importante avec moins de $1 \%$ de superficie soit 11712 ha. Les espèces dominantes sont: Combretum glutinosum, Sterculia setigera, Pterocarpus lucens, etc. Chaque strate identifiée a fait l'objet d'une coloration particulière pour permettre de la singulariser. A ces strates s'ajoutent le réseau hydrographique et routier. On obtient ainsi la carte d'occupation des sols et des types de végétation (Figure 4).

\section{Les différentes zones de la réserve de biosphère}

La réserve de biosphère du Ferlo couvre une superficie totale de 2058214 ha. Elle est subdivisée en trois zones (Tableau 2). Le zonage (Figure 5) s'adapte à différentes formes d'utilisation des terres et est lié aux fonctions de la réserve de biosphère, sans toutefois les superposer. Une seule zone fait l'objet d'une obligation de protection légale ; c'est l'aire centrale. La zone tampon est essentiellement définie par sa contribution aux objectifs de conservation. Enfin l'aire de transition est plus directement consacrée au développement et à des modes d'exploitation durable.

Les aires centrales s'étendent sur 242564 hectares intégralement inclus dans les deux réserves de faune du Ferlo nord et du Ferlo sud. Ces réserves bénéficient d'un statut légal de protection. La Réserve de Faune du Ferlo Nord a été classée par décret $N^{\circ} 72346$ du 21 mars 72 et la Réserve de Faune du Ferlo Sud par décret $\mathrm{N}^{\circ} 72347$ du 21 mars 72. Les aires centrales sont des échantillons d'écosystèmes représentatifs de l'originalité du site et de son intérêt pour la conservation. Elles sont soustraites aux activités humaines, à l'exception des activités de recherche et de surveillance continue, ainsi que, dans certains cas, des activités de collecte traditionnelles exercées par les populations locales.

Les zones tampons qui s'étendent sur 1156633 hectares, sont composées d'habitats d'une grande importance écologique. Elles englobent une partie des 2 réserves de faune (aires centrales exclues), les réserves sylvopastorales de Younouféré, de MBemMBem et de Sabsabré, et la forêt classée de Velingara (Figure 5). Les forêts classées ont un statut juridique et font parties des forêts du domaine national. Dans ces forêts, les populations sont autorisés à exercer des droits d'usages portant sur: le ramassage du bois 
mort et de la paille - la récolte de fruits, de plantes alimentaires ou médicinales, de gomme, de résines de miel - le parcours du bétail, l'émondage et l'ébranchage d'espèces fourragères - la coupe du bois de service destiné à la réparation des habitats. Ces droits sont en conformité avec l'esprit de la zone tampon. Cependant toutes les activités menées dans les zones tampon ne doivent pas aller à l'encontre des objectifs de conservation assignés à l'aire centrale, mais elles doivent au contraire contribuer à la protection de celle-ci.

La zone de transition ou aire de coopération d'une superficie de 659019 hectares, est définie en fonction des activités utilisatrices de ressources naturelles qui s'y mènent. Elle est caractérisée par une grande diversité des systèmes d'utilisation des terres (établissements humains, pâturages, zones de culture, couloirs de transhumance...) et intègre les Unités Pastorales (UP) de
Loumbol, Malandou et Windé Diohi et la réserve naturelle communautaire (RNC) de Mbounguiel.

Les réserves naturelles communautaires (RNC) et les Unités Pastorales (UP) sont des aires communautaires mises en place avec une approche participative et qui ont fait l'objet d'une délibération par le Conseil Rural. Les 4 RNC/UP de la réserve de biosphère sont dotées de plans locaux d'aménagement et de gestion communautaire et de chartes locales de bonne gestion.

La zone de transition est également le lieu privilégié pour la sensibilisation environnementale, l'expérimentation du développement durable et la gestion respectueuse des ressources. Elle a une fonction fondamentale pour la dimension humaine des réserves de biosphère.

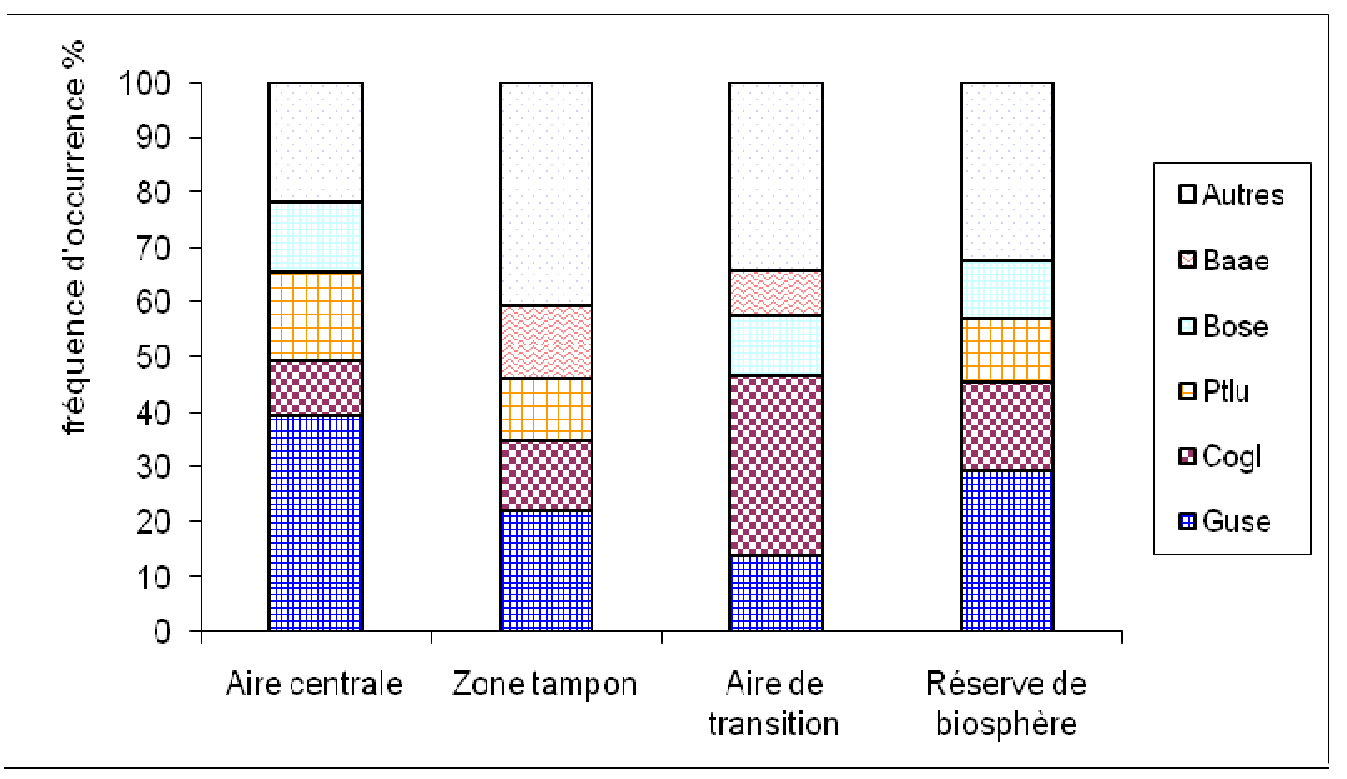

Figure 3: Spectre d'abondance des espèces ligneuses dans la RB du Ferlo. 


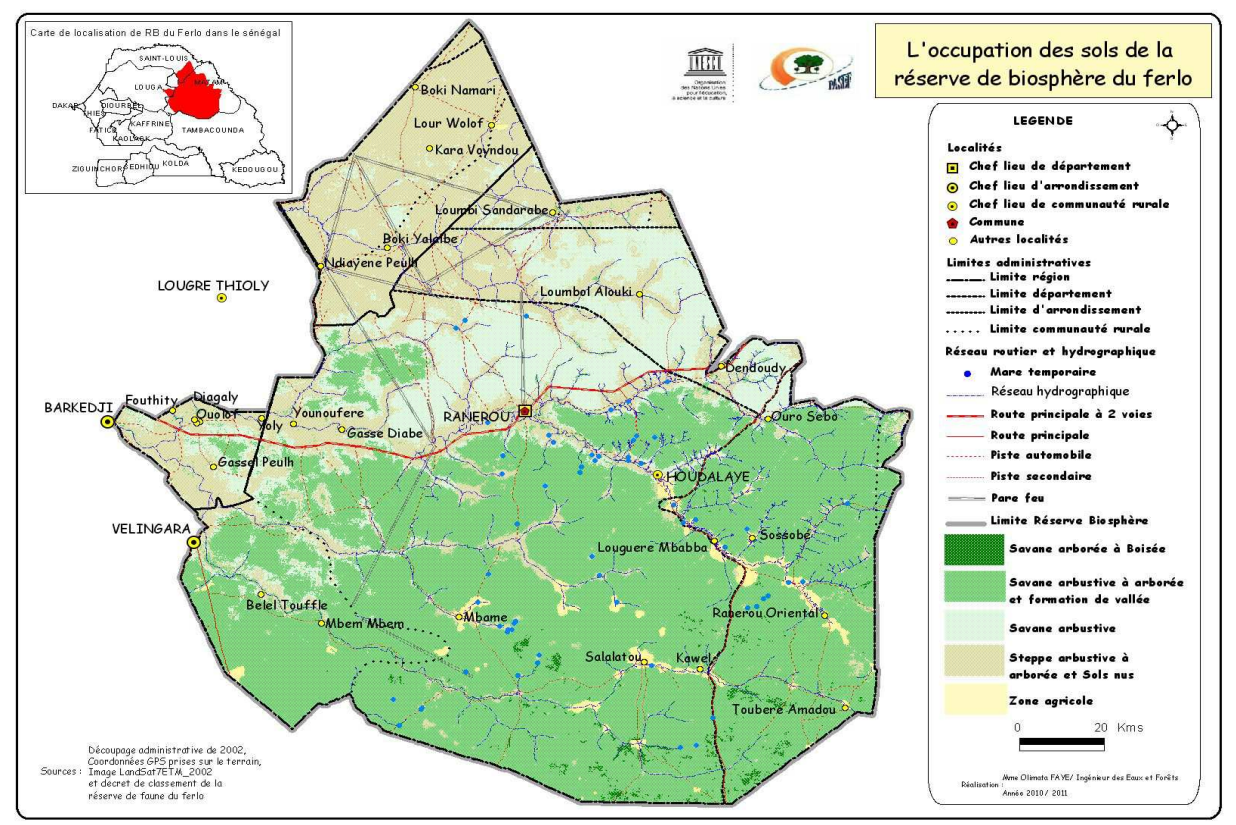

Figure 4: Carte d'occupation des sols de la réserve de biosphère du Ferlo.

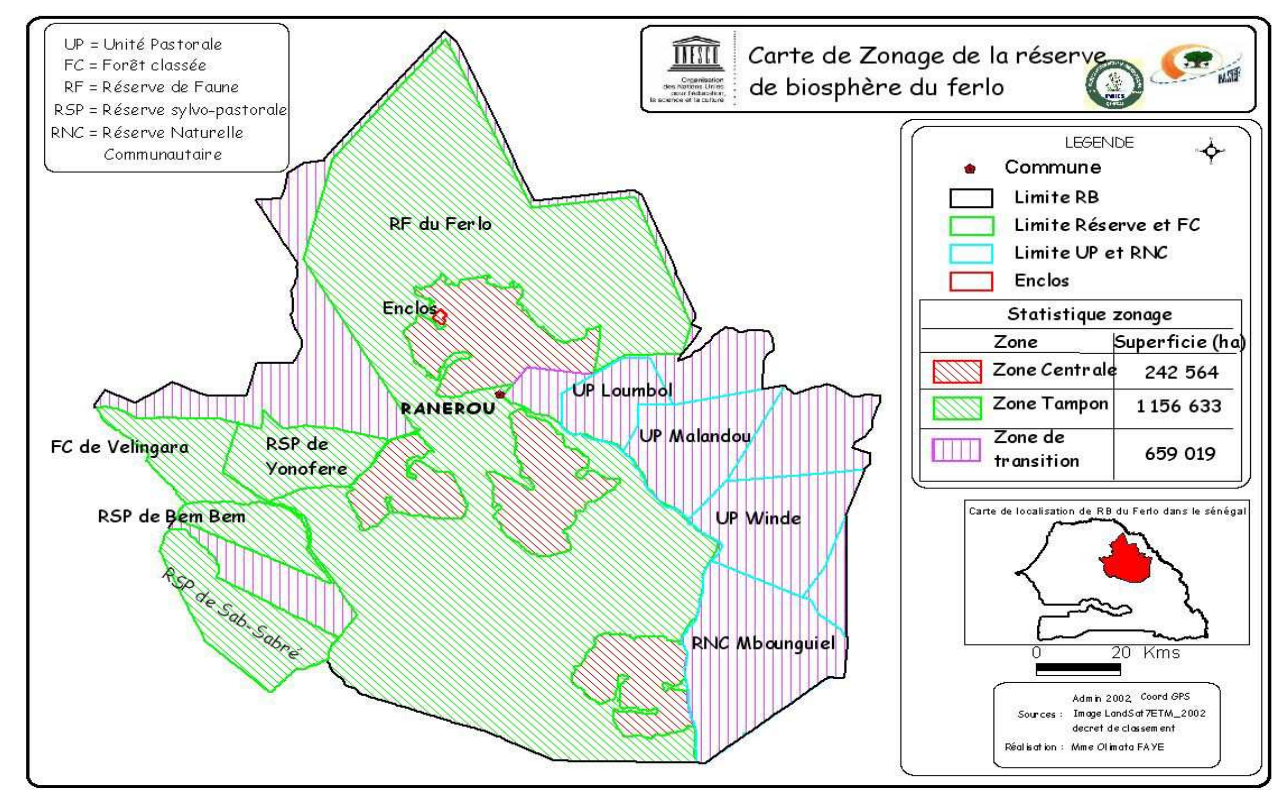

Figure 5: Carte de zonage de la réserve de biosphère du Ferlo. 
Tableau 1: Paramètres structuraux de la végétation ligneuse de la RBF.

\begin{tabular}{lcccc}
\hline Paramètres écologiques & Aire centrale & Zone tampon & Aire de transition & RBF \\
\hline Densité réelle $\left({\mathrm{n} . h \mathrm{~h}^{-1}}^{-1}\right)$ & 392 & 352 & 347 & 389 \\
Taux de couverture \% & $32 \%$ & $43 \%$ & $35 \%$ & $35,30 \%$ \\
Surface terrière $\left(\mathrm{m}^{2} \cdot\right.$ ha $\left.^{-1}\right)$ & 6,1 & 9,17 & 8,64 & 7,44 \\
Taux de régénération \% & $79 \%$ & $36 \%$ & $39 \%$ & $72 \%$ \\
Indice de Shannon & 2,92 & 3,55 & 3,49 & 3,45 \\
\hline
\end{tabular}

Tableau 2: Statistique du zonage de la réserve de biosphère du Ferlo.

\begin{tabular}{lcc}
\hline Zones & Superficies (ha) & Pourcentage \\
\hline Aires centrales & 242564 & 12 \\
Zone tampon ou de connectivité & 1156631 & 56 \\
Zone de transition ou de coopération & 659019 & 32 \\
TOTAL & 2058214 & 100 \\
\hline
\end{tabular}

\section{DISCUSSION}

Le zonage de la réserve de biosphère du Ferlo obéit aux règles de la stratégie de Séville et du cadre statutaire des réserves de biosphère. Il est organisé selon trois zones interconnectées : les aires centrales, protégées par la législation nationale, la zone tampon et la zone de transition. Une caractérisation du peuplement ligneux dans les différentes zones à permis de déterminer les différents paramètres structuraux de la végétation.

La densité d'arbres qui est de 389 pieds/hectare dans l'ensemble de la réserve varie en fonction des différentes zones. Elle est plus élevée dans l'aire centrale (392) que dans la zone tampon (352) et l'aire de transition (347). Cet état de fait s'explique par la plus faible présence de l'homme dans l'aire centrale que dans les autres unités. L'aire centrale qui a la densité la plus élevée de la réserve de biosphère, présente la surface terrière la plus faible parce que la flore est dominée par Guiera senegalensis et Boscia senegalensis qui sont des arbustes, avec des troncs de faible grosseur. Ceci confirme les travaux de Bouxin (1975) selon lesquels il n'existe pas de parallélisme entre la surface terrière et la densité.
Le taux de recouvrement est de 35,3\% dans l'ensemble de la RBF. Ce couvert végétal, plus élevé en zone tampon (43\%) que dans les autres unités, s'explique par la présence d'arbres à grandes cimes (Adansonia digitata, Sterculia setigera, Pterocarpus lucens...).

L'étude du spectre d'abondance des espèces constituant le peuplement végétal présente une grande importance car elle a permis de mieux décrire la diversité que le seul recours à d'autres indicateurs quel que soit leur degré d'élaboration (Ramade, 2003). L'examen du spectre d'abondance des espèces montre que les quatre espèces les plus abondantes sont Guiera senegalensis (29,5\%), Combretum glutinosum (15,9\%), Pterocarpus lucens $(11,6 \%)$ et Boscia senegalensis $(10,5 \%)$. Ces quatre espèces représentent $68 \%$ de l'ensemble des effectifs de RBF et sont également les quatre espèces les plus fréquentes. Ainsi, il $\mathrm{y}$ a une très forte corrélation entre la fréquence centésimale et l'abondance des espèces.

L'indice de diversité de Shannon est assez élevé dans les différentes zones de la réserve de biosphère. Il est plus élevé respectivement en zone tampon, dans l'aire de 
transition et enfin dans l'aire centrale. Ceci reflète une plus grande diversité dans la zone tampon et l'aire de transition que dans l'aire centrale. Cet indice de diversité élevé confirme également l'importance des potentialités écologiques de la zone étudiée (Gning, 2008).

La régénération naturelle est à la base de la compréhension de la dynamique de la végétation ligneuse. Elle peut être végétative ou par semis naturel. Elle passe par le recrutement, la mortalité juvénile et les différents stades de développement, puis la survie (Traoré, 1997). Le taux de régénération du peuplement végétal est de $72 \%$ dans l'ensemble de la RBF. Il est deux fois plus élevé dans l'aire centrale (79\%) que dans la zone tampon $(36 \%)$ et l'aire de transition (39\%). Ceci est lié au fait que l'aire centrale qui bénéficie d'un statut légal de protection subit moins la pression de l'homme que les deux autres zones où des établissements humains sont installés et où tous les systèmes d'utilisation des terres sont rencontrés.

Cette caractérisation des ressources végétales a permis de mieux appréhender le zonage de la réserve de biosphère du Ferlo.

En effet, l'application du zonage varie selon les contextes et implique souvent que la réserve de biosphère soit constituée d'une mosaïque d'aires protégées ayant des statuts plus ou moins contraignants, ainsi que de zones n'ayant pas le statut d'aires protégées et constituant l'aire de transition (Jardin, 2008). Dans la réserve de biosphère du Ferlo, les aires centrales délimitées sont des zones de conservation qui participent du maintien de la biodiversité du milieu, tant des espèces endémiques, menacées et celles sur la liste rouge de l'UICN (année de la référence). Ces aires centrales présentent de nombreux atouts notamment, une superficie de 242564 ha soit $12 \%$ de la superficie totale de la réserve (Ngom, 2011) et des pâturages relativement en abondance en saison pluvieuse. Elles renferment des espèces végétales endémiques et des espèces animales relictuelles d'importance nationale, sous régionale et même internationale. Le Ferlo constitue actuellement le seul refuge des autruches à cou rouge (Struthio camelus Linné) dans la bande sahélienne. Il est aussi un des rares sites au monde à avoir des individus de gazelle à front roux (Gazella rufifrons Gray.) à l'état sauvage (Sow et Akpo, 2011).

L'existence de règles d'accès et d'utilisation rationnelle des ressources pastorales, de même que les aménagements pour la conservation de la faune et de la flore (pare feux, enclos pour les gazelles et les oryx), constituent des atouts supplémentaires pour la conservation des aires centrales.

La zone tampon ou zone de connectivité, qui est attenante à l'aire centrale ou qui l'entoure, (UNESCO, 2004) a pour fonction essentielle de réduire au maximum les effets externes négatifs des activités humaines sur la ou les aires centrales. Outre ce rôle, elle a sa propre fonction intrinsèque, indépendante, de maintien de la diversité biologique et culturelle anthropogénique (UNESCO-MAB, 2008). Ainsi, dans le processus de zonage de la réserve de biosphère du Ferlo, une attention particulière était portée sur la zone tampon où le compromis optimal entre conservation et développement a été recherché. En effet, l'efficacité d'une règle ne doit pas seulement être mesurée au vu de ses effets en termes de conservation mais aussi par le rapport entre ses effets et les contraintes qu'elle impose. Si le même résultat en termes de conservation d'une espèce végétale ou animale est obtenu soit en interdisant l'accès des populations à la zone, soit en autorisant une exploitation raisonnée de ces ressources, la seconde solution sera retenue comme étant plus efficace en termes de coût-bénéfice. La recherche d'un compromis optimal entre conservation et développement passe par la participation des acteurs de base du développement local, qui sont particulièrement bien informés pour savoir comment, en se basant sur un objectif de conservation donné, diminuer les contraintes qu'ils devront supporter (Beuret, 2006). 
Ainsi, le choix de la délimitation d'une zone tampon très vaste (1 $156 \quad 633$ ha), s'inscrit dans une volonté de lui faire jouer une fonction de connectivité, en reliant les éléments de biodiversité des aires centrales à ceux des aires de transition. En effet, dans un contexte spatial étendu, la zone tampon permet une meilleure protection des aires centrales et une meilleure préservation des corridors de migration de la faune de la réserve de biosphère du Niokolo koba vers celle du Ferlo.

La zone de transition ou aire de transition a pour fonction essentielle de contribuer au développement socioéconomique des communautés locales et au développement durable de la réserve de biosphère ainsi que de l'ensemble de sa région. Un des défis de la création et de la gestion d'une réserve de biosphère est de concilier sur un même espace des objectifs de conservation et de développement économique et de faire converger sur le long terme les intérêts des acteurs (MAB, 2003). Dans le processus de zonage de la réserve de biosphère du Ferlo, les préoccupations des communautés locales ont été prises en compte. En effet, les réserves de biosphères servent à aider les populations à améliorer leur bien-être sur le plan économique par le biais de l'utilisation de technologies appropriées. Lorsque ces activités sont choisies par les populations locales, elles leur permettent de gagner leur vie pendant leur séjour dans la réserve et empêchent la destruction des ressources devant être préservées (UNESCO, 1997). Ainsi, la réserve de biosphère du Ferlo constitue une excellente plateforme de concertation et de dialogue entre les différents acteurs, au bénéfice aussi bien de la conservation que du développement.

Aujourd'hui, les réserves de biosphère ne sont plus de simples aires protégées mais des projets d'aménagement du territoire, des lieux d'expérimentation du développement durable et des zones servant de laboratoires pour des chercheurs des différentes disciplines nourrissant les sciences de la conservation au sens large (Cibien et al., 2006). Ce sont des territoires pour l'homme et la nature (MAB France, 2000). La fonctionnalité multiple de la réserve de biosphère du Ferlo et l'intégration des fonctions de conservation, de développement et de support logistique ou de production de savoir dénote une option de durabilité.

La réalisation de ce zonage participatif et fonctionnel de la réserve de biosphère du Ferlo permettra de faciliter le dialogue et la concertation entre les acteurs concernés par l'espace et ses ressources. Cela semble être l'une des voies privilégiées pour gérer la biodiversité dans une optique de développement durable et pour prévenir l'explosion des multiples conflits.

\section{RÉFÉRENCES}

Akpo LE, Grouzis M. 1996. Influence du couvert sur la régénération de quelques espèces ligneuses sahéliennes (Nord Sénégal, Afrique occidental). Webbia 50(2): 247-263.

Arbonnier M. 2002. Arbres, arbustes et lianes des zones sèches d'Afrique de l'Ouest. CIRAD et Museum d'histoire naturel de Paris, 573 p.

Aubreville A. 1957. Accord à Yamgambi sur la nomenclature des types africains de végétation. Bois et Forêts des Tropiques, 51: 23-27.

Berhaut J. 1967. Flore du Sénégal (2 ${ }^{\mathrm{ème}}$ édn). Edition clairafrique: Dakar, Sénégal; 485 p.

Beuret JE. 2006. Dialogue et concertation dans les réserves de biosphère: Problématique et enjeux. In Biodiversité et acteurs: itinéraires de concertation. Rev. Réserves de biosphère, Notes techniques 1- 2006, 9-21.

Boudet G. 1984. Manuel sur les Pâturages Tropicaux et les Cultures Fourragères (4 ${ }^{\text {ème }}$ édn). Ministère de la Coopération, Manuel et Précis d'Elevage: Paris ; 254 p.

Bouxin G. 1975. Ordination and classification in the savana vegetation of the Akagera Park (Rwanda, Central Africa). Vegetation, 29: 155-157. 
Cibien C, Bioret F, Genot JC. 2006. Mettre en œuvre le concept de réserve de biosphère a l'échelle du territoire: diversité des structures et des acteurs. In Biodiversité et acteurs: itinéraires de concertation. Rev. Réserves de biosphère, Notes techniques 1- 2006 : 22-24.

Cornet A. 1992. Relation entre la structure spatiale des peuplements végétaux et le bilan hydrique des sols de quelques phytocénoses en zone aride. In L'Aridité une Contrainte au Développement. Editions ORSTOM; 245-263.

Faye O. 2011. Cartographie de la réserve de biosphère du Ferlo. Rapport de consultance, $26 \mathrm{p}$.

Frontier S. 1983. L'échantillonnage de la diversité spécifique. In Statégie d'Echantillonnage en Ecologie. Editions Frontier et Masson: Paris (Coll. D'Ecologie), XVIII + 494 p.

Frontier S, Pichod-Viale D. 1998. Ecosystèmes: Structure, Fonctionnement, Evolution (2 ${ }^{\text {ème }}$ édn). Dunod: Paris; 447 p.

Gning ON. 2008. Caractéristiques des ligneux fourragers dans les parcours communautaires de Khossanto (Kédougou, Sénégal Oriental). Mémoire de $D E A$ en Biologie végétale, UCAD, $78 \mathrm{p}$.

Gounot M. 1969. Méthodes d'études quantitatives de la végétation. Masson et Cie: Paris.

Jardin M. 2008. Le cas particulier des réserves de biosphère. In Entre l'homme et la nature, une démarche pour des relations durables Réserves de biosphère. Notes techniques $\mathrm{N}^{\circ} 3,32-36$.

Long G. 1974. Diagnostic Phyto-Ecologique et Aménagement du Territoire. Principes Généraux et Méthodes. Masson: Paris, $252 \mathrm{p}$.

MAB. 2003. Développement du Réseau mondial de réserves de biosphère: Proposition d'une stratégie du MAB pour la prévention et la résolution des conflits dans les réserves de biosphère. Document de travail. Bureau du MAB, 8-11 juillet 2003. SC-03/CONF.217/6.
MAB France. 2000. Réserves de biosphère, des territoires pour l'Homme et la Nature. Octavius Gallimard. Gallimard Jeunesse, Paris.

Magurran AE. 1988. Ecological Diversity and its Measurement, Princeton NJ. Princeton University Press; 179 p.

Ngom D. 2011. Formulaire de proposition de la réserve de biosphère du Ferlo. Rapport de consultance. $75 \mathrm{p}$.

Ramade F. 2002. Dictionnaire Encyclopédique de l'Ecologie et des Sciences de l'Environnement (2 $2^{\mathrm{ème}}$ édn). Dunod: Paris; 1075 p.

Ramade F. 2003. Eléments d'Ecologie: Ecologie Fondamentale ( $3^{\text {ème }}$ édn). Dunod: Paris ; 690p.

Sow AA, Akpo LE. 2011. Plan de coopération de la réserve de biosphère du Ferlo. Rapport de consultance, 51p.

Traoré SA. 1997. Analyse de la flore et de la végétation de la zone de Simenti (Parc National du Niokolo Koba), Sénégal Oriental. Thèse de $3^{\text {ème }}$ cycle. FST/UCAD, Sénégal, 136 p.

UNESCO. 1996. Réserves de biosphère: la Stratégie de Séville et le cadre statutaire du réseau mondial. UNESCO, Paris, 23p.

UNESCO. 1997. MAB en Afrique : rétrospectives et perspectives pour le $21^{\text {ème }}$ siècle. UNESCO-Dakar, 23p.

UNESCO. 2003. Réserves de biosphère: des lieux privilégiés pour les hommes et la nature. UNESCO, Paris, 208p.

UNESCO. 2004. Explique-moi... Les Réserves de biosphère. UNESCO, Paris, $39 \mathrm{p}$.

UNESCO. 2006. Biodiversité et acteurs: itinéraires de concertation. Rev. Réserves de biosphère, Notes techniques 1- 2006, $82 \mathrm{p}$.

UNESCO. 2008. Le plan d'action de Madrid pour les Réserves de biosphère. UNESCO, Paris, 37p. 